\title{
Provide a Way to Reduction of Energy Consumption in Wireless Sensor Networks using the concepts of Fuzzy Logic and Genetic Algorithm.
}

1Sayyed Hedayat Tarighi Nejad,2 Reza Alinaghian 3- third author.

1 .computer college, Islamic Azad University of Najaf Abad.

2. .computer college, Islamic Azad University of Mobarakha.

3 . Position or scientific order, respective university or organization of third author.

\begin{abstract}
Wireless sensor networks are a collection of small sensor nodes that can monitor and sense of their surroundings and sending data to a main station. The limited energy of nodes is a major challenge of sensor networks that affect the survival of the network. Thus, as yet is presented several methods to optimization of energy consumption and increasing the lifetime of a sensor network. In this paper, using fuzzy system design and system optimization by genetic algorithm is presented approach to select the best cluster head in sensor networks. Using random data set has been addressed to evaluate of fuzzy-genetic system presented in this paper and finally, MSE rate or mean error of sending the messages using proposed fuzzy system in comparison with LEACH method is calculated in select the cluster head. The results of evaluations is representative of a reduction the MSE metric in proposed method in comparison with LEACH method for select the cluster head. Reduce of MSE directly is effective on energy consumption and lifetime of wireless sensor network and can cause the reduction of energy consumption and increase the lifetime of the networks.
\end{abstract}

Keywords: wireless sensor networks, fuzzy logic, fuzzy system, genetic algorithm, energy consumption, clustering.

\section{Council for Innovative Research}

Peer Review Research Publishing System

JOURNAL: INTERNATIONAL JOURNAL OF MANAGEMENT \& INFORMATION TECHNOLOGY

Vol. 10, No 9

editorsijmit@gmail.com

www.jjmit.com 


\section{Introduction}

With the advent and development of microelectronic technology in the late 70s, the new sensors were considered. Using microelectronic technology, were produced the low-cost sensors with small dimensions and low weight. New raw materials for fabrication of the sensor is discovered, and subsequently considered the new principles for practical purposes and data collection. The integration of sensor and electronic circuitry of signal anamorphic is created significant opportunities for the majority of applications. Today, reduce the size and weight of the sensors and increases their susceptibility is the main goal of many research laboratories and different companies. The small size of sensor nodes was sense of smaller their energy productive batteries. The main reason for the emergence and development of wireless sensor networks has been applications of continuous monitoring of environments that achieve and permanent human presence in them is difficult or impossible. Applications such as monitoring the eruption of active volcano, monitoring the battlefield or sensitive regions of military and etc. As a result, generally recharging or replacing the dead nodes (disabled due to the finish of the energy source)is not possible, because as was said these nodes usually placed in the environments and hard, rough and inaccessible conditions and often become scatter randomly and appropriately in environment. So two points are important in the performance of sensor networks: one is the lifetime and another is network coverage of these networks. Since monitoring applications are typically time-consuming, it is expected that the lifetime of sensor networks to be long enough. But if we total space the network divide into virtual areas, usually in each area there are several sensor node. Thus, in case of death some of nodes an area, other nodes can be partially maintain the network coverage. But if die all nodes of placed in the area of network, virtually is impossible the area monitoring and called will disappear the network coverage. Therefore, random and scattered death of sensor nodes, is better than aggregation the dead nodes in the area. Therefore, the solution that considered for achieve to increase lifetime and maintain the network coverage in sensor networks, is reduction of energy consumption of nodes, simultaneous with energy uniform consumption in all nodes in the network. With respect to these reasons, the main purpose of sensor networks is wise and rational management of energy resources. Therefore, it is first necessary to understand the resources of energy consumption as well. Some of the energy consumptions of sensor node may be useful, such as 1-sending or receiving data 2-processing queries of requested 3sending queries and data to neighbor nodes. But, use of high and idle of energy in sensor node related to: 1.passive listening: that is listening to the inactive channel for a possible traffic receiving.2. Collision (clash): that is when a node receive more than one package at a time, even if receive two packages are only partially synchronic. All package that are cause clash, must be discarded and were de novo sending, that the retransmission cause increase of energy consumption

3. Eavesdropping: that is a node the receive the packages that are sent to other nodes.4.overhead of control packages: thus, the minimum control packages should be used for sending data.5.send a message while the destination node is not ready to receive[1]. It is necessary to manage the energy consumption that we know of energy consumption level of different of the sensor node. The following points are noteworthy about the energy consumption of various parts a sensor node, that have a important role in the application of the reduction of energy consumption. As shown in figure1, the communication subsystem than the computing subsystem has a much higher energy consumption. It is proven that send a bit of information can consumption the energy in extent the execution of thousands of instructions. Therefore, a compromise must be established between the communication and the processing.

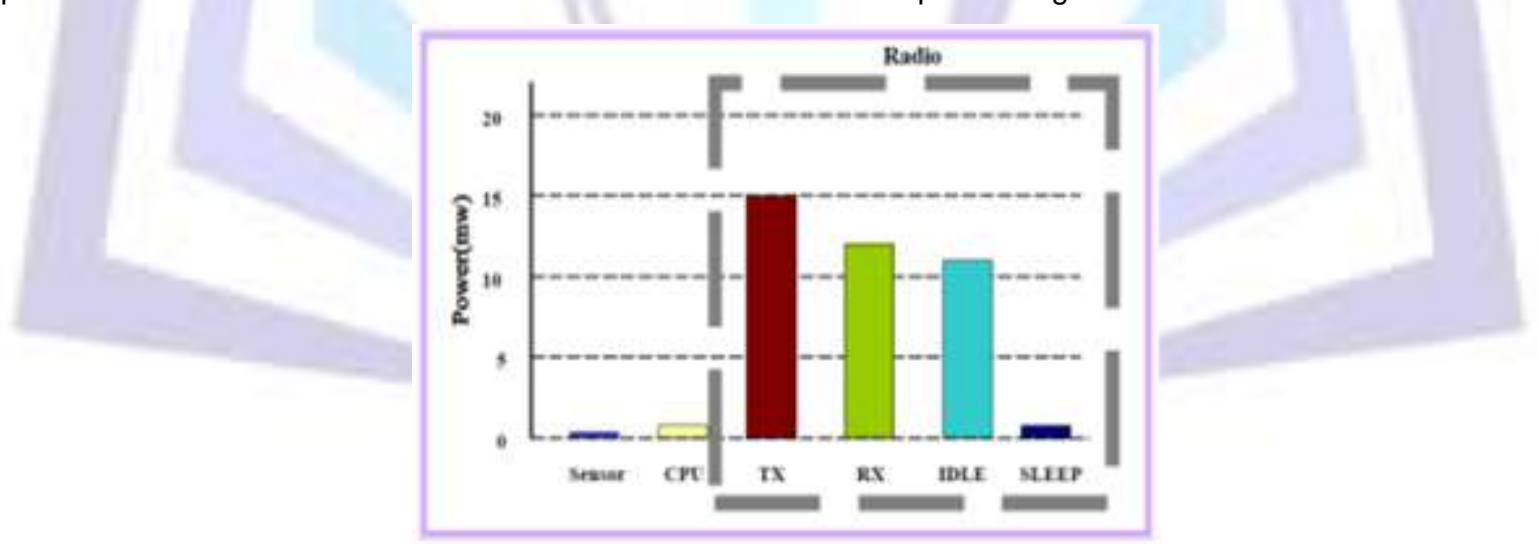

Figure 1. comparison of energy consumption in different parts of the sensor node.

Energy consumption in conditions of reception, transmission and idle is almost the same size (figure1). At the same time,energy consumption of radio below fall at least one order of magnitude in sleep mode. Therefore, the radio should whenever possible stand in sleep (idle) mode.

According to the specific application, sensory subsystem may be a significant source of energy consumption. Therefore, energy consumption must be reduced. The most important problem this is that to minimize the energy consumption of sensor nodes, at the same time we meet the needs of the application. Today, the power dynamic management techniques that attention to the reduction of energy consumption of sensor networks after their design and placement, had been addressed the greatest attention and have received many research studies around the world. Also, in recent years, the possibility of using intelligent tools such as neural networks has been proved in ways to the increase of energy efficiency in wireless sensor networks. 
Despite accomplished advances in this type of networks, sensor nodes due to the large number, small size and placement of the contingent, still to meet its energy are based on batteries with low power. Also, due to the use of such networks in harsh environments and inaccessible, there is on possibility of recharging or replacing the sensor nodes. Therefore, one of the most important issues in wireless sensor networks is problem of a server energy limitation. Also, since the sensor network efficiency is depend heavily on network lifetime and its network coverage, so in terms of energy storage algorithms in design of sensor networks with long life is vital. In recent years was created the strong tendencies to use artificial intelligence techniques to the reduction of energy consumption in wireless sensor network. For example, artificial neural networks due to the possess of convenient features such as automatic classification of data the reduction of data dimensions, easy processing almost in all aspects of the reduction of energy consumption in wireless sensor networks are used as effective and powerful tool[2]. As shown in figure1, communications in wireless sensor networks are one of most use the energy consumption cases. In this paper with studies conducted for problem of cluster-head selection in wireless sensor networks and using the uncertainties in the properties of each node at different times, we have proceed for fuzzy system design[3]to select the best cluster-head in every moment. For the proposed fuzzy system design and optimization of provided fuzzy system parameters, was used genetic optimization algorithm 4]. The proposed fuzzy system is evaluated on the designed data set to evaluate of energy consumption level in sensor networks and the results is calculated to determine the reduction of energy consumption in the proposed method. The obtained results of cluster-head selection in wireless sensor networks using proposed fuzzy system in comparison with cluster-head selection method of LEACH, is representative of the reduction of energy consumption in the proposed method of study. Reduction of energy consumption in wireless sensor networks is effective directly on the performance and the increase of longevity of networks and can be considered an important step to achieve QOS in sensor networks. In the second part of this article has been addressed to review new recent studies of carried out in the cluster-head selection for wireless sensor network and also carried out studies to design of fuzzy systems using innovative algorithms and particular the genetic algorithm. In the third part is described the fuzzy system and in part IV has been addressed to examine the data set of used in the evaluation and also analysis of the obtained results to determine the reduction of energy consumption by using the proposed fuzzy system. In the final part also has been addressed to present the conclusion and recommendation for more studies in this field of research.

\section{RELATED WORKS}

For the clustering in wireless sensor networks, is presented many algorithms that below refer to some of them. LEACH algorithms[5] which is most known as the clustering algorithm, select the cluster head node by a random process. The algorithm the act fully distributed and when a node the selected as a cluster head node, all nodes with select of the closest node to the cluster head node the placement in formed clustered. In this algorithm may in a period unselected any cluster head or to be select many number of cluster head. Another problem that there are in this method is that may all the cluster head to be select in the angle of network. The most important problem of LEACH is switching overhead of clusters and energy dissipation versus other algorithms. In algorithm of LEACH-C[6]which is an improved LEACH, each node the sent the location and the amount of remaining energy to SINK then sink, nodes of the cluster head the choose by a central algorithm. Furthermore in order to better understand the proposed methods for clustering and selection of cluster head in wireless sensor networks has been addressed to classify these methods in table 1.

Table(1): the most important proposed methods for clustering and selection of cluster head in wireless sensor networks.

Table(1)

\begin{tabular}{|c|c|c|c|}
\hline year & disadvantages & Proposed method & method \\
\hline 2002 & $\begin{array}{c}\text { Substitution overhead of } \\
\text { clusters and loss energy }\end{array}$ & $\begin{array}{l}\text { Cluster head node the } \\
\text { selection in form of a } \\
\text { random process. }\end{array}$ & LEACH [6] \\
\hline 2002 & $\begin{array}{c}\text { Is very sensitive and dependent } \\
\text { on SINK position }\end{array}$ & $\begin{array}{c}\text { SINK the select to cluster } \\
\text { head nodes by central } \\
\text { algorithm }\end{array}$ & LEACHECES[7] \\
\hline 2009 & $\begin{array}{c}\text { Clusters are fixed and have not } \\
\text { very application dynamic } \\
\text { sensor networks }\end{array}$ & $\begin{array}{c}\text { Divide the network into } \\
\text { several parts with equal size } \\
\text { and then classification these } \\
\text { parts to clusters with } \\
\text { different sizes }\end{array}$ & PEBEC \\
\hline 2009 & $\begin{array}{c}\text { The increase of information } \\
\text { dispatch rate }\end{array}$ & Use of multi-level clustering & ESC [8] \\
\hline
\end{tabular}

Then, according to the concepts of artificial intelligence such as fuzzy and genetic system in proposed method, using intelligent ways has been addressed to review the most important carried out studies for clustering and selection of cluster head in wireless sensor networks. For example, shiyuan jin using genetic algorithm, dynamically select the clusters and cluster heads, but in this method, fitness function only is considered the distance between the nodes and cluster head number and regardless of the remaining. But since the cluster heads the consumption the amount of more energy than conventional nodes, so one of the main parameters for the selection of the cluster heads is the amount of energy remaining of sensors. 
In table(2) using the concepts of artificial intelligence has been addressed to the most important proposed methods for clustering and selection of cluster head in wireless sensor networks and disadvantages of each of these methods are also investigated.

Table (2) : the most important proposed methods for clustering and selection of cluster head in wireless sensor networks using the concepts of artificial intelligence.

Table(2)

\begin{tabular}{|c|l|l|c|}
\hline year & \multicolumn{1}{|c|}{ disadvantages } & \multicolumn{1}{|c|}{ Proposed method } & method \\
\hline 2008 & $\begin{array}{l}\text { Only is considered } \\
\text { distance between } \\
\text { nodes and number } \\
\text { of cluster head and } \\
\text { regardless of } \\
\text { residual }\end{array}$ & $\begin{array}{l}\text { Using genetic algorithm, } \\
\text { clusters and cluster heads the } \\
\text { select to dynamicaly }\end{array}$ & Shiyuan Jin[9] \\
\hline 2005 & $\begin{array}{l}\text { In each period be } \\
\text { select only a } \\
\text { cluster head }\end{array}$ & $\begin{array}{l}\text { Is used fuzzy logic for find } \\
\text { cluster heads }\end{array}$ & Gupta [10] \\
\hline 2002 & $\begin{array}{l}\text { The creation of the } \\
\text { less number of } \\
\text { cluster }\end{array}$ & $\begin{array}{l}\text { LCA the select a node as } \\
\text { cluster head that have more } \\
\text { characteristic than neighbor } \\
\text { cluster head }\end{array}$ & LCA[11] \\
\hline
\end{tabular}

Fuzzy logic is presented for the first time in 1965 by professor Zade and so far have been great advances[12]. Of the most important studies carried out for fuzzy logic can refer to present of fuzzy system by Mamdani and sugeno[13]. In order to design the optimal fuzzy system also was carried out studies among 14] and [15], that in each of them using innovative algorithms such as genetic and particle swarm optimization algorithm has been addressed to offer of methods for design the optimal parameters in the fuzzy system.

\section{Presentation of the proposed method}

In this section, by review of proposed methods to discuss challenges in this study and with the use of fuzzy system we have to choose the best node of cluster head in sensor network.

In 1-3 and 2-3 sections due to the use of fuzzy logic and genetic algorithm, in proposed method of paper has been addressed to study the basic concepts. In section 3-3, using LEACH algorithm has been addressed to examine of how to choose the cluster head in sensor networks, and in section 4-3 has been addressed to description of the proposed method to reduction the energy consumption in wireless sensor networks.

\section{3-1. fuzzy logic}

Uncertainty has been always one of the challenges of very important in different science. In recent years, researchers using study and recognition of uncertainty, the provide methods and models for the implementation of uncertainty in engineering sciences that result of them is offer of concepts such as fuzzy logic. Including the most modern advances in engineering systems is presentation and implementation of fuzzy system for control process. In fuzzy system that with two types of Mamdani and sugeno[13], using the concepts of fuzzy Logic, fuzzy inference, absolute numbers and fuzzy set to study the environment and uncertain decision, we have to make decision to solve problems[16.] In figure 2, has been addressed to examine the most important components in design the fuzzy system of sugeno that used in this study[17].

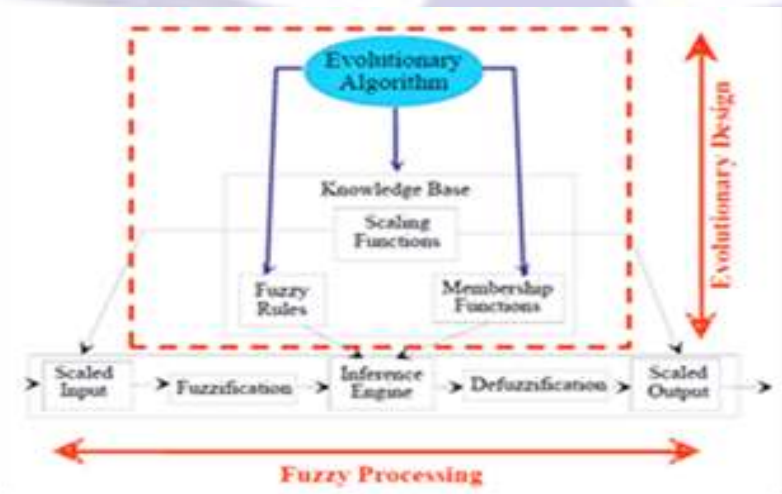

Figure2: study of most important components in design the fuzzy system of sugeno

The most important components used in the fuzzy system, is part of the knowledge base, that using fuzzy rules and fuzzy logic to determine for fuzzy decision-making. To understand how decision-making in inference engine of fuzzy system in figure (3), using fuzzy rules and terms and linguistic variable has been addressed to graphically to evaluate how fuzzy inference[18]. 
1.
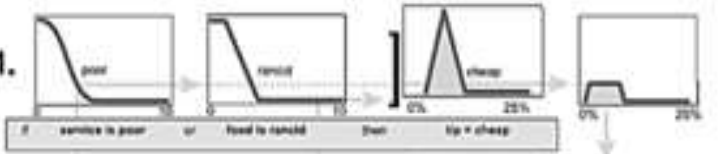

2.
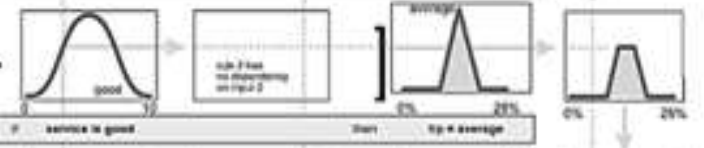

3.

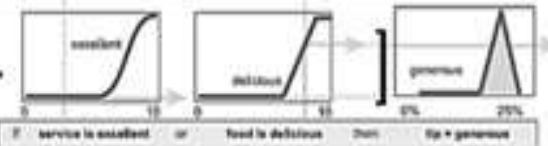

r.

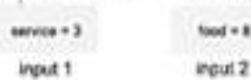

incui 2

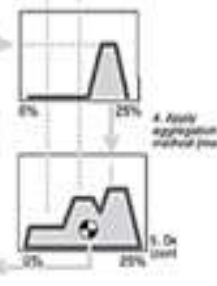

oupu

Figure3: study of fuzzy inference using fuzzy rules in the system.

In the above figure using fuzzy operators of MAX-MIN has been addressed to fuzzy inference for three rules mentioned in figure[3]. The several types of fuzzy function there are for use in fuzzy system that with regard to problem, use of these functions. For example, membership degree in fuzzy function of bell-shaped is like figure (4)[18].

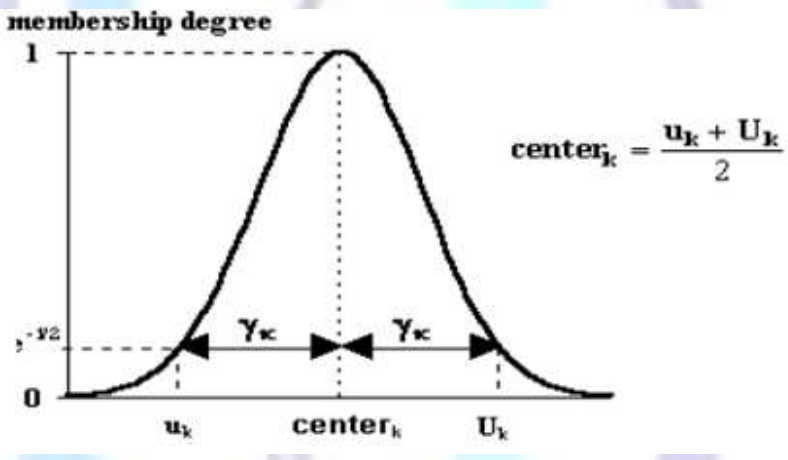

Figure (4): membership degree in fuzzy function of bell-shaped.

As indicated in figure 2, for design a fuzzy system is require to several parameters including optimal rules. For design the optimal parameters in design the fuzzy system, presented different methods that in this study is used the genetic algorithm.

In next section has been addressed to a short presentation of the basic concepts of this algorithm.

\section{2-3. Genetic algorithm}

GA algorithm is a developed search method by Holland 1975[19], which is consistent based on the mechanism of natural selection and reproduction to a random search but oriented through decision making area for find the optimal solutions of comprehensive. Basically, GA algorithms of system to provide feasible solutions for the optimization need that usually feasible solution to be define by a linear chain called chromosome that its length is different in terms of the type application. In GA systems occur three basic functions: reproduction, exchange selection and mutation.

Reproduction (proliferation) is process in which chains in terms of weight factor that is depend on optimal of solutions of chain (fitness function) were reproduce.

Exchange is process in which new chain randomly combined together and each chain pair (parent) the exchange part of its information and the creation a new chain pair (child). Mutation is process which by it alteration the amount of one of chain rings randomly. Each chain ring (box) is an indicator of gen. Number above the box is indicator of activity status in the network (activity number) and numbers inside the boxes are for the indicator of option number for activity.

In the process of exchange, two members randomly selection, and their chromosomal information be exchange. Exchange is done only with exchange with p-c possibility. It is said that the power of GA is in exchange process in which occur random exchange the genetic characteristics, that probably a good answer can make a good answer. 
In figure 2, chains of 1 and 2 randomly selection, in a random point (gene 5) has been broken, was exchange the genetic information and shaped are two new chains.

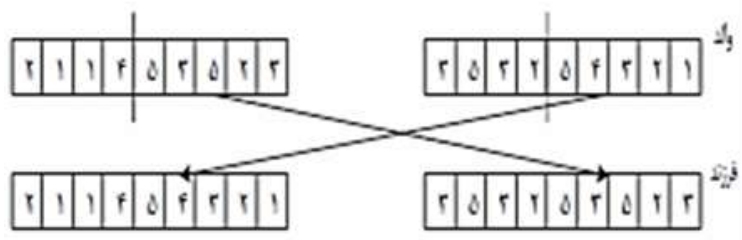

Figure 5: exchange operation

In mutation process, change the genetic contents of some of the genes of an answer (with probability pm). GA system socontinue with reproduction, exchange and mutation process. After several generations is achieved optimal solution. Thebalance between exchange and mutation is accomplishable with possibility their occurrence. Commonly,

$$
\begin{aligned}
& { }^{*} \leq \leq P_{e} \leq 1 \\
& * * 1 \leq P_{m} \leq * *
\end{aligned}
$$

The following using a genetic algorithm has been addressed to describe how proposed system design and optimization of fuzzy system rules.

\section{3-3. cluster head selection algorithm of LEACH}

Clustering hierarchy protocol of adaptive with low energy is first and most well-known protocol based on clustering in wireless sensor networks, in which occur the creation of clusters in the form of distributed. The most important purpose of $\mathrm{LEACH}$ is have a local base stations (cluster head) for reduction the energy consumption result of data transmission to a remote base station. LEACH the chosen a small sensor node randomly as cluster head and organization the local nodes as local clusters. Assign of nodes to respective cluster head is done based on proximity(distance). Non-cluster head nodes(also called normal nodes), transfer the data to cluster head. Therefore, the only overhead that there are for them, is intra-cluster communication. Cluster head nodes than normal nodes the need to more energy. Therefore, selecting of fixed cluster head nodes lead to early energy discharge and their premature death. The balance of energy of cluster heads with periodic rotation the role of the cluster head is established between the different nodes. Also, the use of data collection or composition in the cluster heads, lead to reduction the message transfer to the base station and resulting in energy storage. LEACH protocol operation is divided into several rounds. Each round begin with installation stage(cluster form), where clusters are organize. Following the installation stage, there is data transfer stage in which normal nodes, send the data to the cluster heads and cluster heads after data collection or composition process, transfer the integrated packing into the base station until reduce the amount of data that must send to base station. In LEACH, timing of sensor data transmission is performed by protocol of code Division Multiple Access(CDMA) or time division multiple Access(TDMA). Select the cluster head is done through a probability function. Each node the select a random number between zero and one, and if the selected number is less than $T(n)$, that node is selected as current round cluster head(5).

Formula(1)

$$
T(n)=\left\{\begin{array}{l}
\frac{P}{1-P\left(r \bmod \frac{1}{P}\right)} \\
0
\end{array}\right.
$$

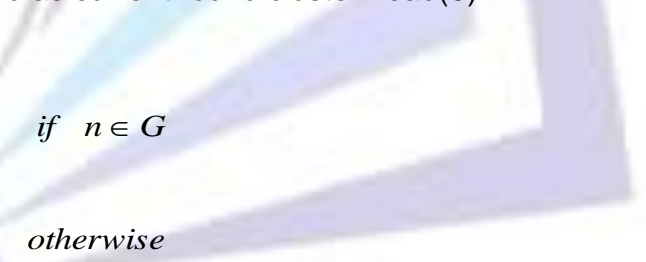

$P$ is the probability of the cluster head, $r$ is the current round number and $G$ is set of nodes of that in current round $1 / p$ were not a cluster head. Based on simulation model, has been shown that only five percent of the clusters are require to getting cluster head. The strength point of LEACH is in the rotation mechanism the role of the cluster heads and data collection also extend the lifetime of the network.

\section{4-3. Describe the proposed method}

To solve the problem of choosing the best cluster head in wireless sensor networks, in this paper the control method is presented based on fuzzy logic. In the proposed method that in figure 6 is a schematic of it visible, at first, all nodes to send the message. After forward of the messages, the residual energy of each node and the distribution of each node the available of the proposed fuzzy control. The proposed fuzzy control using the residual energy of each node and the distribution of each node, proceed to the decision making to select the best cluster head in sensor network. Linguistic variable represent the energy of a node in sensor networks, energy level and distribution of a number of neighbors of a node. 


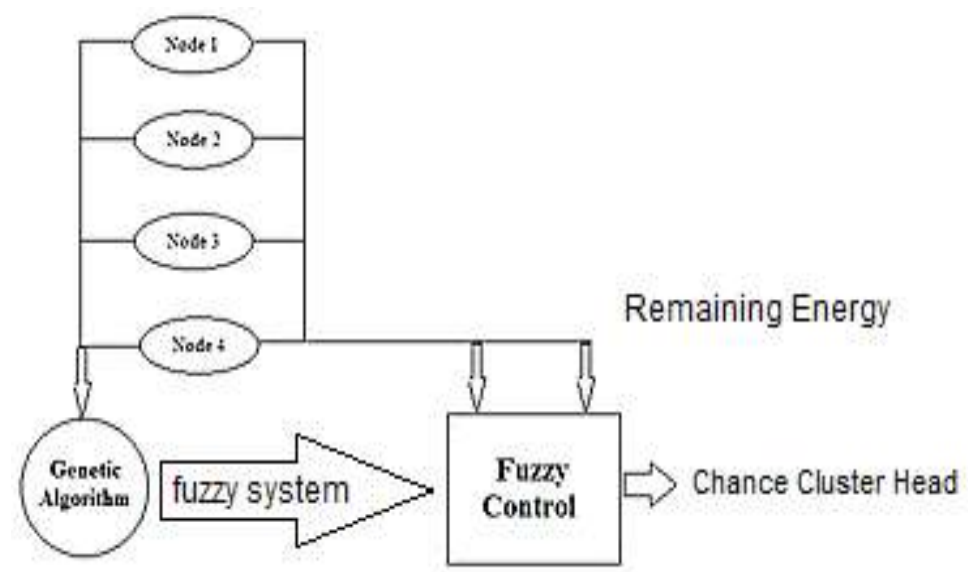

Figure(6): schematic of application stages of the proposed method to select the best cluster head in wireless sensor networks.

Using proposed method in this research, first, error level of transmission of messages by any of the nodes in a sensor network the send to designed genetic algorithm and genetic algorithm using sensing in formation will attempt to fuzzy system construction and extract the fuzzy rules. Then, fuzzy control using the remaining energy level in each node and the distribution of each node will attempt to the optimal cluster head and proportion with capability of each node. To used fuzzy system design in proposed method, is necessity to the extraction of optimal parameters, which as explained, in this paper was used genetic optimize algorithm. Procedures of forenamed algorithm in this paper is that at first, error level of transmission of messages by any of the nodes in a sensor network the send to genetic algorithm as input. Then, the algorithm using conversion of fuzzy rules into the chromosomes and algorithm genes will attempt to determine the best rules in the proposed fuzzy system and with the production of different generations and with the rules will be produced in each generation, will reduce the error rate in the selection the cluster head.

In figure (7) has been addressed to determine the mentioned cases for fuzzy system optimization and fuzzy rules.

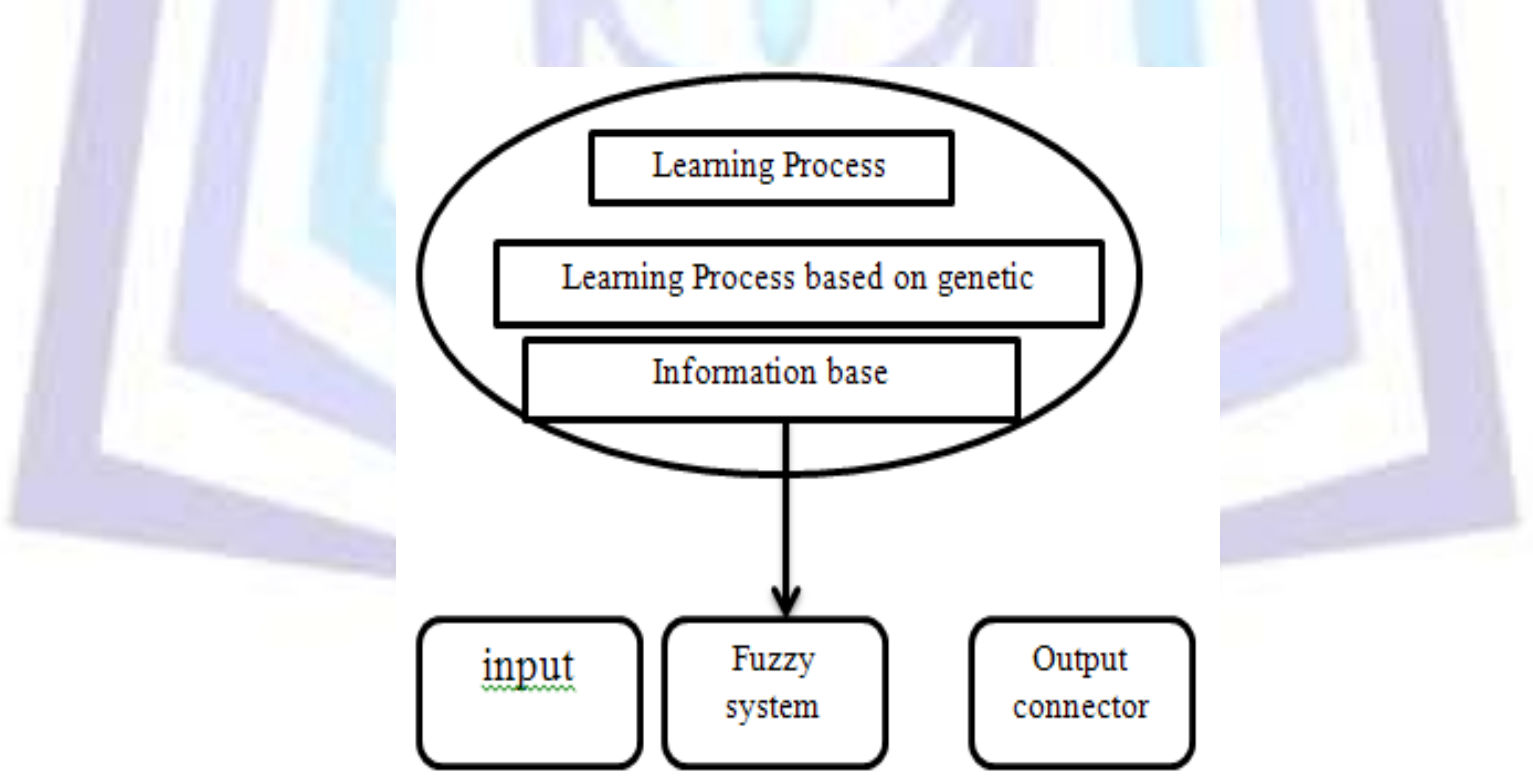

Figure7: flowchart the optimization of fuzzy rules in the proposed fuzzy system.

Among the most important parameters in the design of genetic algorithm used in this study, may be cited to cost function. For calculate the cost function in this study, is used squared error metric(formula3) in energy consumption of sensor networks. Then, in figure 8 is visible the overview of proposed fuzzy system for selection the cluster head in sensor networks which is simulated in MATLAB software. 


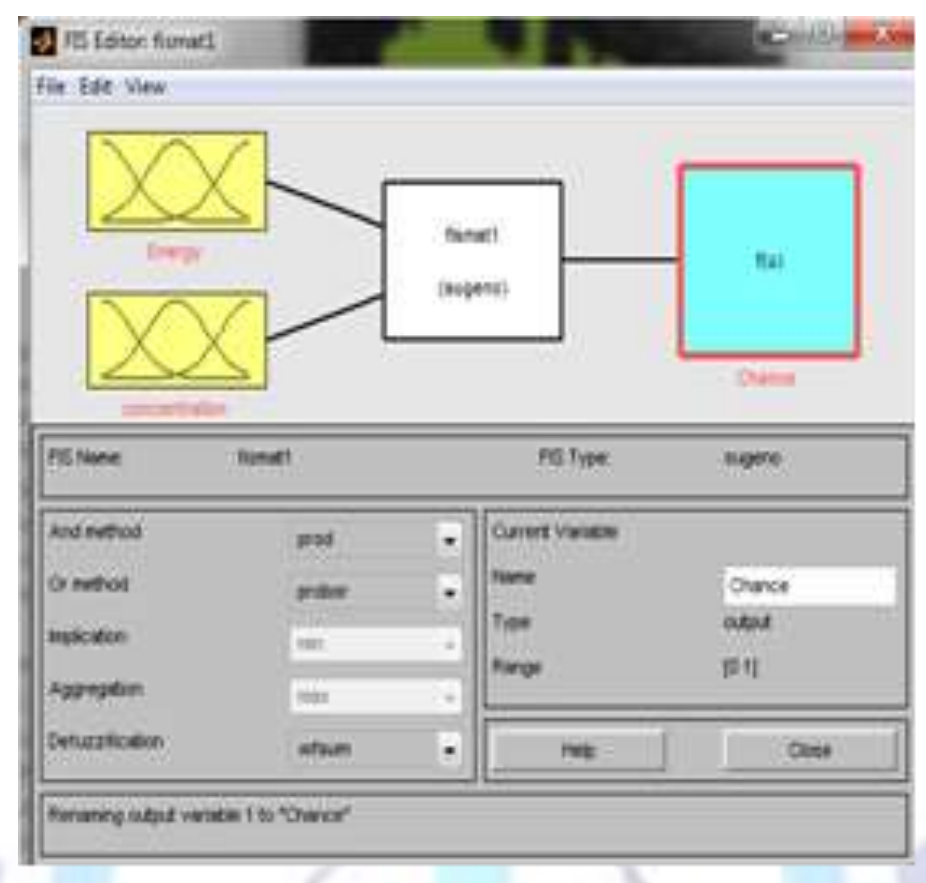

Figure8: the overview of proposed fuzzy system for selection the cluster head in sensor networks.

Finally for better understanding, a summary of the parameters used in used genetic algorithm, is presented in table3.

\begin{tabular}{|c|c|c|c|c|c|c|}
\hline Mutation & $\begin{array}{c}\text { Population } \\
\text { size }\end{array}$ & Selection & $\begin{array}{c}\text { Crossover } \\
\text { fraction }\end{array}$ & Elite count & Generations & Fitnees \\
\hline gaussian(0.1,0.1) & 25 & stochastic uniform & 8 & 2 & 75 & SE \\
\hline
\end{tabular}

Table3: The most important parameters used in the genetic algorithm to optimize of proposed fuzzy system rules. After the fuzzy system rules be optimization, rules designed in the inference engine of proposed fuzzy system will be used for selection the of best cluster head in sensor networks, that in figure 9 designed inference engine is visible for proposed fuzzy system.

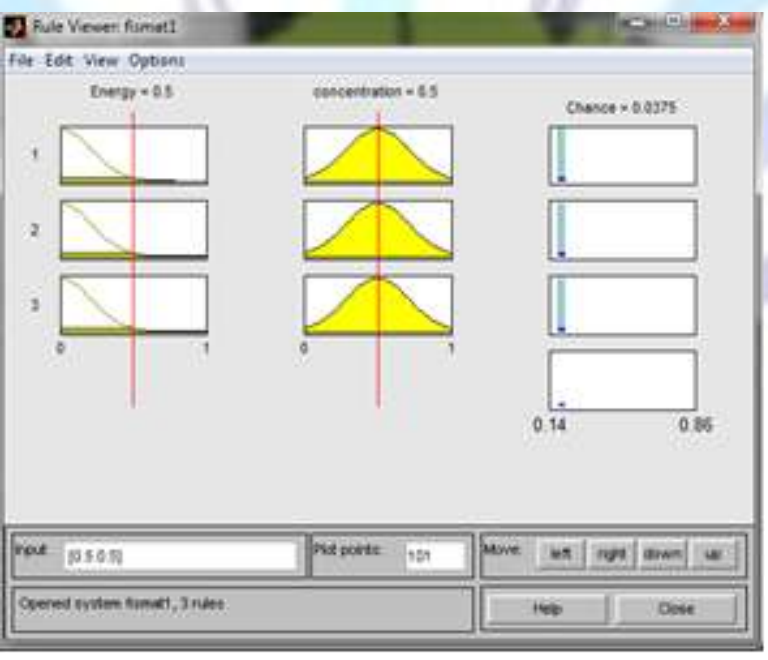

Figure9: The designed inference engine for proposed fuzzy system.

In figure 10 has been addressed to examine how to reduce the error rate in the rules used in the proposed fuzzy system that are designed by the genetic algorithm. 


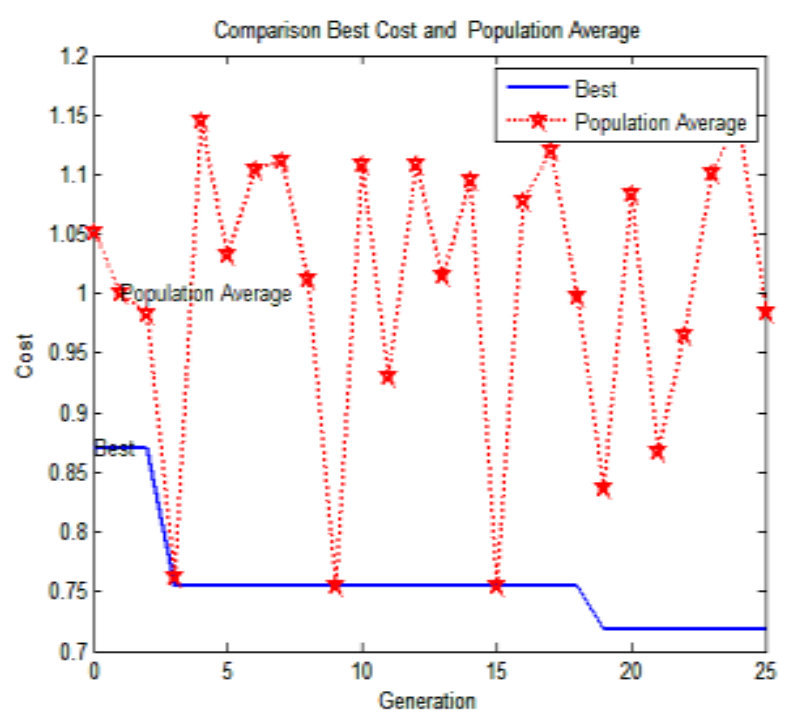

Figure10: The reduce the error rate in different generations used in the genetic algorithm.

In this example using production of 25 generations we have been able achieve to the nearest value to zero the error rate in proposed fuzzy system rules for selection of the best cluster head in wireless sensor networks. The reduce the error rate in the selection of the best cluster head in wireless sensor networks will be lead to reduction the energy consumption in a time period and less energy in sensor networks is to sense of extend the lifetime of this type of network. In the next section to evaluate the proposed method in this paper, has been addressed to present used data set in the evaluation and as well as the presentation and analysis of the evaluation results for reduction of energy consumption in sensor networks.

\section{Evaluation of results}

In this section, has been addressed to determine and evalution of proposed method to reduction of energy consumption in sensor networks.

In section 1-4 is presented used data set in the evaluation of research, in section 2-4 has been addressed to describe the evaluation method and finally section 3-4 is presented analysis of evaluation results.

\section{1-4. Data set of evaluation}

To evaluation of proposed method using MATLAB software is studied for fuzzy system simulation. As mentioned in the previous section, to use of proposed method at first must be available the proposed system the amounts of features of residual energy level in each and also distribution the each node.

In conducted studies in this study, for the design of the proposed fuzzy control is need to consider the most important factors of effect on selection the node as cluster head, that have important role in the probability of getting cluster head a node in sensor network[20]. Other considered factor in this study is scattering, that factor have also closely relation with the probability of getting cluster head a node in sensor networks, and further dispersion each node is in sense of less chance that node for creation the cluster head[20].

Using the formula of conditional probability, can calculate the probability of getting cluster head each of the nodes based on the amount of energy and dispersion each node. Also, using ratio creation can determine the probability of getting cluster head each node than increase or decrease each of the factors of energy and dispersion. In this study, for gather of training data set and assessment of proposed fuzzy system, we use the ratio. In the following is presented the necessary formula for the collection of research data.

\section{Formula(2): $Z=X / Y$}

In formula(2), $z$ is the probability or chance of getting cluster head each node, $x$ is the energy of each node and $y$ is the dispersion of the node in sensor network. Because by increasing the amount of energy of each node should increase the probability of getting cluster head that node, so it has been the value of $\mathrm{X}$ in the numerator and because by increasing the amount of dispersion of the each node should decrease the probability of getting cluster head that node, so it has been the value of $Y$ in the denominator.

To evaluate the proposed method we have attempted to design a hypothetical example of a sensor network. In this designed example, the message the use of 4 cluster head to send messages and the amount of messages is 400 messages, that is presented in table(4) assumptions and characteristics of each node in the designed example 
Table(4): primary data set for evaluation the proposed method.

\begin{tabular}{|c|c|c|c|c|}
\hline $\begin{array}{c}\text { The percent of } \\
\text { actual fitness of } \\
\text { getting cluster } \\
\text { head }\end{array}$ & $\begin{array}{c}\text { The percent of } \\
\text { the probability } \\
\text { of getting } \\
\text { cluster head in } \\
\text { the LEACH } \\
\text { method }\end{array}$ & $\begin{array}{c}\text { The percent of the } \\
\text { probability of } \\
\text { dispersion }\end{array}$ & $\begin{array}{c}\text { The percent of the } \\
\text { residual energy }\end{array}$ & $\begin{array}{c}\text { The nodes of } \\
\text { sensor network }\end{array}$ \\
\hline 30.33 & 28 & 0.15 & 0.50 & Node 1 \\
\hline 7.5 & 11 & 0.20 & 0.15 & Node 2 \\
\hline 17.5 & 28 & 0.20 & 0.35 & Node 3 \\
\hline 12.5 & 0.40 & 0.5 & Node 4 \\
\hline
\end{tabular}

In the next section using the designed example in this section and using the proposed fuzzy system has addressed to explain the evaluation method used to assess the reduction of energy consumption in sensor networks.

\section{2-4.Describe the evaluation method}

To evaluate the proposed method, at first using presented data set in the previous section that is considered as input of proposed fuzzy system, has been addressed to measure the probability of getting cluster head each of the nodes in designed example by the proposed fuzzy system. The obtained results is visible in the table(5).

The SE is calculated from absolute value of the difference between the percent of actual fitness of getting cluster head and the percent of the probability of getting cluster head in any way. The equation of require for the calculation of ES in the form below.

Formula(3):

$\mathrm{SE}=\mid$ the percent of the probability of getting cluster head in any way - the percent of real fitness of getting cluster head|

Table (5):SE value for each selected node by proposed fuzzy system.

\begin{tabular}{|c|c|c|c|c|}
\hline $\begin{array}{l}\text { The nodes of } \\
\text { sensor network }\end{array}$ & $\begin{array}{l}\text { The percent of the } \\
\text { residual energy }\end{array}$ & $\begin{array}{l}\text { The percent of the } \\
\text { probability of dispersion }\end{array}$ & SE & \begin{tabular}{l} 
The percent of $\begin{array}{c}\text { The } \\
\text { probability of getting cluster } \\
\text { head taken into account by } \\
\text { proposed fuzzy system }\end{array}$ \\
\hline 1
\end{tabular} \\
\hline 2 & 0.50 & 0.15 & 2.33 & \\
\hline 3 & 0.15 & 0.20 & 25.5 & 25 \\
\hline 4 & 0.35 & 0.20 & 6.5 & 20.5 \\
\hline
\end{tabular}

After extraction the percent of the probability of getting cluster head of each node in the simulation of proposed fuzzy system, then has been addressed to evaluation of designed messages in four nodes. To compare the probability of getting of getting cluster head in proposed fuzzy system and LEACH method in the table (6), the message transmission is calculated using both method. 
Table(6): the compare the message taken into account in the proposed method and LEACH method for designed example and research evaluation.

\begin{tabular}{|c|c|c|c|}
\hline node & $\begin{array}{c}\text { The number } \\
\text { forwarding } \\
\text { messages in } \\
\text { proposed fuzzy } \\
\text { method }\end{array}$ & $\begin{array}{c}\text { The number forwarding } \\
\text { messages in LEACH } \\
\text { method }\end{array}$ & $\begin{array}{c}\text { The number of actual messages } \\
\text { in terms of fitness each node }\end{array}$ \\
\hline 1 & 128 & 112 & 121.32 \\
\hline 2 & 102 & 132 & 70 \\
\hline 3 & 82 & 44 & 50 \\
\hline 4 & 88 & 112 & 28 \\
\hline
\end{tabular}

As is visible in the above, the deviation rate in the number of message in the proposed method in all nodes comparison with LEACH method, is less ratio the number of actual messages of require in terms of fitness each node. In the next section using the calculation of SE value in the proposed method and LEACH method, proposed fuzzy system has been addressed to calculate the reduction of energy consumption in sensor networks.

\section{3-4. Analysis of results}

Using the obtained results from the above, in this section has been addressed to calculate the time of forwarding messages at four nodes that are 400 messages. According to the results the table(6), SE value in the proposed method and LEACH method for selection cluster heads is calculated in hypothetical example of sensor network and is provided in the table(7).

Table (7) : the time of forwarding messages in the proposed method in comparison with LEACH method.

\begin{tabular}{|c|c|c|}
\hline $\begin{array}{c}\text { The } \\
\text { sensor } \\
\text { network } \\
\text { nodes }\end{array}$ & $\begin{array}{c}\text { SE in LEACH } \\
\text { method }\end{array}$ & $\begin{array}{c}\text { SE in proposed fuzzy } \\
\text { method }\end{array}$ \\
\hline 1 & 93.32 & 6.68 \\
\hline 2 & 5 & 74 \\
\hline 3 & 59 & 18 \\
\hline 4 & 22 & 33 \\
\hline total & 179 & 131.68 \\
\hline
\end{tabular}

As is observed in figure(11), according to the obtained results of the average error of forwarding messages because of the death of nodes in the designed example to evaluate the proposed method, in the proposed method and LEACH method is observed that the MSE rate in the proposed method is lower than LEACH method in the selection cluster heads.

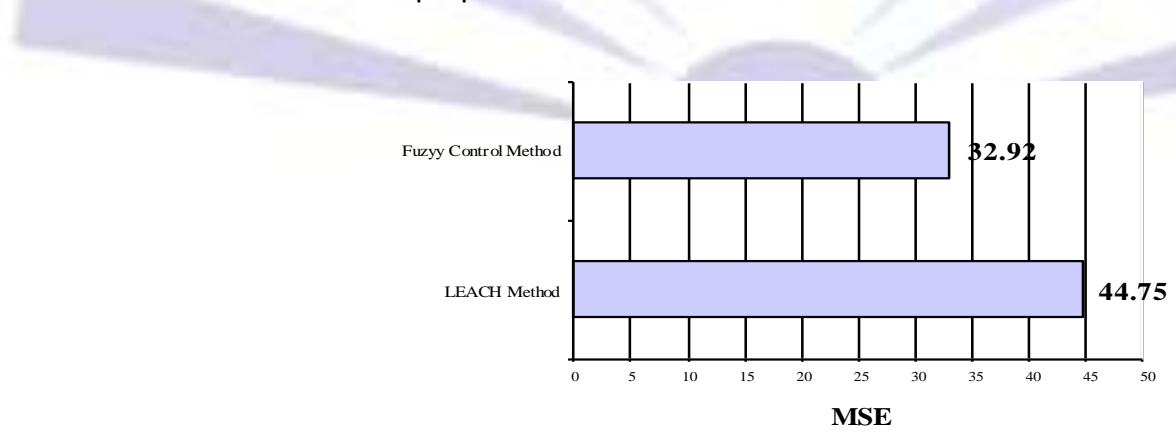

Figure (11): the MSE value in the proposed method in comparison with LEACH method for select the cluster heads.

MSE metric reduction in the proposed method is meant to reduce the error of forwarding messages because of less death of nodes, that the reduction finally will be a lot the amount of energy for sensor network. The reduction of energy consumption of the sensor networks in the proposed method, in turn, increase the lifetime of the network to select the cluster heads in the proposed method in comparison with LEACH method. 


\section{Conclusion}

Given the mentioned challenge in this study that is how to choose the best cluster heads in the wireless sensor networks, in this study has been addressed to provide a control way based on the use of genetic fuzzy systems. In the proposed method, the selecting cluster heads is done by proposed genetic fuzzy system and based on the properties of each node in the moment of forwarding messages that it may help to distribution of cluster heads in form of more balance in sensor networks. To research evaluation, the proposed method is simulated in MATLAB and using design of a hypothetical example in the use of sensor networks has been addressed to determine the MSE rate or mean error of forwarding messages in the proposed method in comparison with LEACH method to selection the best cluster heads. The results the study is indicative of MSE metric reduction in the proposed method in comparison with LEACH method in the selection of the cluster heads. The MSE metric reduction in the proposed method is meant to reduce the error of forwarding messages that the reduction finally will be a lot the amount of energy for sensor network. The reduction finally will be a lot the amount of energy for sensor network. The reduction of energy consumption of the sensor networks in the proposed method, in turn, increase the lifetime of the network to select the cluster heads in the proposed method in comparison with LEACH method.

\section{Reference:}

[1].Anastasi G, Conti M, Passarella A, "Energy Conservation in Wireless Sensor Networks: a survey", Ad Hoc Networks, Volume 7, Issue 3, pp.537-568, 2009

[2].Enami N, Askari Moghadam R. , "Energy Based Clustering Self Organizing Map ProtocolFor Extending Wireless Sensor Networks Lifetime and Coverage", Canadian Journal on Multimedia and Wireless Networks, Vol. 1, No. 4, pp. 4254, 2010.

[3].Dubois, D. and H. Prade, "Fuzzy Sets and systems: theory and applications", Academic Press, New York,1980.

[4].Mukhopadhyay, D. M., Balitanas, M. O., Alisherov F. A., Jeon, S. H. And Bhattacharyya, D., "Genetic algorithm: a tutorial review", International Journal of of Grid and Distributed Computing,Vol.2, No.3, pp.25-32, September 2009.

[5].O. Zzitoune, M . El aroussi ,M. Rziza,D. Aboutajdine, "Stochastic Low Energy Adaptive Clustring Hierarchy", ICGSTCNIR,Vol. 8, Issue. 1, pp. 47-51, 2008

[6].W.B. Heinzelman, A.p.chandrakasan and H.balakrishnan, " "An Application Specific Protocol Arthitecture for Wireless Microsensor Networks ", IEEE Transaction On Wireless Comunication,pp. 660-670, 2002.

[7].J Y.Wang, T.L.X Yang, D.Zhang ,, "An Energy Efficient and Balance Hierarchical Unequal Clustering Algorithm for Larg Scal Sensor Network", Inform. Technol.J.28-38,8(1),2009.

[8].SIM, K .Jin Choi, K Kwon and Jaiyong Lee,, "Energy Efficient Cluster Header Selection Algorithm in WSN ", in proceedings off IEEE international Conference on Complex,Intelligent and Software Intensive Systems,pp. 584-587 2009

[9].Shiyuan Jin , Ming Zhou and Annie S.Wu, "Sensor Network Optimization Using a Genetic Algorithm", School Of EECS,University of Central Florida,Orland ,FL 32816,2008.

[10].Gupta, D Riordan and S. Sampalli, "Cluster- Head Election Using Fuzzy Logic for Wireless Sensor Networks ,"In Proceedings of IEEE Communication Networks and Services Research Conference,pp. 255-260 2005.

[11].W. Ye, J. Heidemann, and D. Estrin, "An Energy - Efficient MAC Protocol for Wireless Sensor Networks", in proceeding 0 the 21st International Annual Joint Conference of the IEEE Coputers and Communication Societies ( INFOCOM 2002 ), New Yok, NY, USA,2002.

[12].Zadeh, L.A., "Fuzzy sets", Information and Control,Vol. 8, pp. 338-353, 1965.

[13].Takagi, T. , Sugeno, M., "Fuzzy identification of systems and its applications to modelling and control", IEEE Trans. Syst. Man Cybern,Vol.15, No.1, pp.116-132, Doi. 10.1109/TSMC.1985.6313399, ISSN. 0018-9472, 1985.

[14].Prasad Reddy, P.V., "Particle swarm optimization in the fine-tuning of fuzzy software cost estimation models", International Journal of Software Engineering (IJSE), Vol.1, No.2, 2009.

[15].Pham, D.T. And Karaboga, D., "Optimum design of fuzzy logic controllers using genetic algorithm", Journal of Systems Engineering,Vol.1, pp.114-118, 1991

[16].Zadeh, L.A., "Knowledge representation in fuzzy logic", IEEE Transactions on Knowledge and Data Engineering,Vol. 1, pp. 89-100, 1989.

[17].Herrera, F. , "Genetic fuzzy systems: taxonomy, current research trends and prospects", Springer,Vol.1, pp.27-46, Doi/10.1007.s12065-007-0001-5, 2008.

[18] Ross, T. J. , Fuzzy logic with engineering applications, John Wiley \& Sons Ltd, University of New Mexico, USA 2004.

[19].Hen'era, F., Lozano, M. And Verdegay, J.L., "A learning process for fuzzy control rules using genetic algorithms", Fuzz Sets and Systems, Vol.100, pp.143-158, Doi.0165-0114/98/\$19.00, 1998.

[20].Shiyuan Jin , Ming Zhou and Annie S.Wu, "Sensor Network Optimization Using a Genetic Algorithm", School Of EECS,University of Central Florida,Orland ,FL 3.2816,2008 